\title{
Numerical investigation of low-velocity filtration combustion instability based on the initial preheating non-uniformity
}

\author{
Yongfang Xia, Tingyong Fang, Haitao Wang, Erbao Guo, Jinwei Ma \\ Anhui Jianzhu University \\ ${ }^{1}$ School of Environment and Energy Engineering, Hefei, 230009 China \\ ${ }^{2}$ Key Laboratory of Huizhou Architecture in Anhui Province, Hefei, 230009 China
}

\begin{abstract}
The effects of the initial preheating perturbation on the dynamical behaviors of FGC wave propagation instability for low-velocity FGC in packed bed are studied numerically. The behaviors of the flame front inclination, break, and shrinking instabilities are always observed in experiments. Based on the experimental phenomena, an initial thermal perturbation model is numerically proposed as to predict the deformation behaviors of the flame front instabilities. The typical flame shapes are obtained depending on filtration velocity, equivalence ratio, and initial preheating temperature difference. It is demonstrated that the development of flame front inclination instability is proportional to the magnitude of initial preheating perturbation. At a lower equivalence ratio, the initial thermal perturbation of $300 \mathrm{~K}$ leads to the evolution of flame front break. Increasing filtration velocity leads to the appearance of flame front break, due to the intensification of the hydrodynamic instability. In addition, a perculiar instability of flame front shifting is also confirmed with the initial thermal perturbation of $400 \mathrm{~K}$, which results in a fuel leakage of incomplete combustion.
\end{abstract}

\section{Introduction}

The inclinational instability of filtration gas combustion (FGC) wave propagation frequently occurs in inert porous media. The inclination instability manifests that the slope of the plannar flame front will occur when it is subject to a small perturbation. For the application of porous media technology, this instability is negative factor, which may lead to unstable operation of devices. Thus, considerable investigations of low-velocity FGC instability have been conducted by many researchers for the last several decades.

The FGC stability with respect to spatial perturbations in packed bed was investigated theoretically, experimentally by Minaev et al. [1]. It was shown that the filtration wave propagation is hydrodynamically unstable if the reactor diameter of burner exceeds a critical value. Meanwhile, they analyzed the dependence of the FGC wave inclinational angle on both the gas-filtration rate and mixture compositions. Debrego et al. [2, 3] experimentally, analytically and numerically studied the thermalhydrodynamic dynamics of FGC wave front inclination instability in a packed bed. The concept of perturbation two-staged evolution, which includes initial linear perturbation growth due to local filtration redistribution and following complex thermal and hydrodynamic reorganization of the system, is proposed. It was found that the inclination amplitude growth velocity on the initial linear stage is proportional to the filtration combustion wave velocity, the reactor system diameter, and the inversed diameter of porous media particles.In addition, Zheng et al., Shi et al. [4, 5] studied experimentally, numerically the effects of variation of different parameters on the inclinational angle growth of FGC flame front distortion.

In the present study, some instabilities of lowvelocity FGC in a packed bed with alumina particles are investigated numerically. The main objectives are to manifest the characteristics of several wave propagating instabilities for low-velocity FGC, which are induced by initial temperature non-uniformity at the preheating section of burner. A simple perturbation model of initial preheating non-uniformity is established in order to quantitatively confirm the possible mechanism of the FGC wave propagation instabilities. For various inlet filtration velocities and equivalence ratios, several combustion wave instability regimes are divided into several regions of existence in plot for the initial setting preheating temperature difference of $300 \mathrm{~K}$.

\section{Mathematical model}

\subsection{The physical model of burner}

Two-dimensional physical model of burner is assumed as shown in Fig. 1, which is completely the same with the experimental burner in geometrical size, including the inlet section with length of $50 \mathrm{~mm}$, the initial

\footnotetext{
$\overline{\text { * Corresponding author: yongfangxia@ } 126 . c o m}$
} 
preheating section with length of $50 \mathrm{~mm}$, and the combustion wave propagation section with length of 400 $\mathrm{mm}$. The initial preheating section is simply divided into two equivalent zones, in which the perturbation of various initial preheating temperatures difference may be set as the ignition temperature range of $1400 \sim 1700 \mathrm{~K}$.

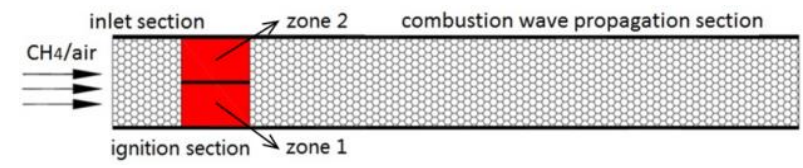

Fig. 1. Schematics of burner model with a simplified ignition temperature difference model.

\subsection{Governing equations}

In order to simplify the mathematical model, some assumptions need to be done. The gas mixture is ideal gas, and the gas radiation is neglected. The porous media material is homogeneous, inert, and optically thick. The scattering of burner wall is also neglected. The Dufour/Soret thermal diffusivity effects and buoyancy effect is not considered. Additionally, the volumeaveraged treatment is considered, since the real perturbation of local porosity non-homogeneity is neglected in this paper. A set of equations are expressed as follows.

(1) Continuity equation

$\frac{\partial\left(\varepsilon \rho_{\mathrm{g}}\right)}{\partial t}+\nabla\left(\varepsilon \rho_{\mathrm{g}} \overrightarrow{\mathrm{u}}\right)=0$

where $\varepsilon, \rho_{\mathrm{g}}, \overrightarrow{\mathrm{u}}$ are the packed bed porosity, the gas mixture density, the velocity vector, respectively.

(2) Momentum equation

$$
\frac{\partial\left(\varepsilon \rho_{\mathrm{g}} u_{i}\right)}{\partial t}+\nabla\left(\varepsilon \rho_{\mathrm{g}} u_{i} \cdot \overrightarrow{\mathrm{u}}\right)=-\frac{\partial p}{\partial x_{i}}+\nabla\left(\mu \nabla u_{i}\right)-\frac{\Delta p}{\Delta L}
$$

where the pressure loss due to the porous matrix is calculated using the following expression.

$\frac{\nabla p}{\nabla L}=180 \frac{(1-\varepsilon)^{2}}{\varepsilon^{3}} \frac{\mu u_{i}}{d^{2}}+1.8 \frac{1-\varepsilon}{\varepsilon^{3}} \frac{\rho_{\mathrm{g}} u_{i}|\mathrm{u}|}{d}$

(3) Gas phase energy conservation equation

$\varepsilon \frac{\partial\left(c_{\mathrm{g}} \rho_{\mathrm{g}} T_{\mathrm{g}}\right)}{\partial t}+\varepsilon \nabla\left(c_{\mathrm{g}} \rho_{\mathrm{g}} \overrightarrow{\mathrm{u}} T_{\mathrm{g}}\right)=\varepsilon \nabla\left(\lambda_{\text {eff: } \mathrm{g}} \nabla T_{\mathrm{g}}\right)+h_{\mathrm{v}}\left(T_{\mathrm{s}}-T_{\mathrm{g}}\right)-\varepsilon \sum_{i} w_{i} h_{i} W_{i}$

where $c_{\mathrm{g}}$ is the specific heat capacity of gas mixture, $h_{\mathrm{v}}$ is the volumetric convective heat transfer coefficient between gas and solid phases, and its expression is $h_{\mathrm{v}}=\left(6 \varepsilon / d^{2}\right) N u \lambda_{\mathrm{g}}$, where $N u$ is described as $N u=2+1.1 \operatorname{Pr}^{1 / 3} \operatorname{Re}^{0.6}[6], \lambda_{\text {eff-g }}$ is the effective thermal conductivity of gas phase and its calculated expression is $\lambda_{\text {eff-g }}=\lambda_{\mathrm{g}}+\rho_{\mathrm{g}} C_{\mathrm{g}} D_{\|}^{d}$, here, $D_{\|}^{d}$ is the thermal dispersion [7], $W_{i}$ is the molecular weight, $w_{i}$ is the reaction rate of the $i^{\text {th }}$ species.

(4) Solid phase energy conservation equation

$$
(1-\varepsilon) \frac{\partial\left(c_{\mathrm{s}} \rho_{\mathrm{s}} T_{\mathrm{s}}\right)}{\partial t}=\nabla\left(\lambda_{\text {eff-s }} \nabla T_{\mathrm{s}}\right)+h_{\mathrm{v}}\left(T_{\mathrm{g}}-T_{\mathrm{s}}\right)
$$

where $\lambda_{\text {eff-s }}$ is the effective conductivity of porous matrix and its expression is $\lambda_{\text {eff-s }}=(1-\varepsilon) \lambda_{\mathrm{s}}+\lambda_{\text {rad }}$, where $\lambda_{\text {rad }}$ is the equivalent radiative conductivity of alumina particles, and its expression is $\lambda_{\text {rad }}=\frac{32 \cdot \sigma \cdot d \cdot \varepsilon \cdot T_{s}^{3}}{9 \cdot(1-\varepsilon)}$, decided by the Rosseland approximation method [8].

(5) Species transport equation

$\varepsilon \frac{\partial\left(\rho_{\mathrm{g}} Y_{i}\right)}{\partial t}+\varepsilon \nabla\left(\rho_{\mathrm{g}} \overrightarrow{\mathrm{u}} Y_{i}\right)=\nabla\left(\varepsilon \rho_{\mathrm{g}} D_{i} \nabla Y_{i}\right)+\varepsilon w_{i} W_{i}$

(6) Ideal gas equation of state

$p=\rho_{\mathrm{g}} R T_{\mathrm{g}}$

\subsection{Boundary conditions}

Inlet condition:

$T_{\mathrm{g}}=300 \mathrm{~K}, u_{\mathrm{g}}=u_{\mathrm{g}, \mathrm{in}}, v=0, Y_{\mathrm{CH}_{+}}=Y_{\mathrm{CH}_{\mathrm{H}}, \mathrm{in}}, Y_{\mathrm{O},}=Y_{\mathrm{O}_{2}, \mathrm{in}}$

The solid phase temperature at the inlet can be given by the following formula.

$\lambda_{\mathrm{s}} \frac{\partial T_{\mathrm{s}}}{\partial x}=-\varepsilon_{\mathrm{r}} \sigma\left(T_{\mathrm{s}}^{4}-T_{\mathrm{o}}^{4}\right)$

Outlet condition:

$\frac{\partial T_{\mathrm{g}}}{\partial x}=\frac{\partial T_{\mathrm{s}}}{\partial x}=\frac{\partial Y_{\mathrm{CH}_{4}}}{\partial x}=\frac{\partial Y_{\mathrm{O}_{2}}}{\partial x}=0$

The solid phase temperature at the outlet can be decided by the following formula.

$\lambda_{\mathrm{s}} \frac{\partial T_{\mathrm{s}}}{\partial x}=-\varepsilon_{\mathrm{r}} \sigma\left(T_{\mathrm{s}, \text { out }}{ }^{4}-T_{\mathrm{o}}^{4}\right)$

Reactor wall condition:

$\lambda_{\mathrm{s} 1} \frac{\partial T_{\mathrm{s} 1}}{\partial y}=h_{\mathrm{air}}\left(T_{\mathrm{ex}}-T_{\mathrm{o}}\right)+\varepsilon_{\mathrm{s} 1} \sigma\left(T_{\mathrm{ex}}{ }^{4}-T_{\mathrm{o}}^{4}\right)$

\subsection{Solution method}

A simplest ignition model set to a preheating section length of $50 \mathrm{~mm}$ with an ignition temperature range of $1400-1700 \mathrm{~K}$. One single global chemistry mechanism of methane/air is applied in this paper. The set of equations is calculated using the Fluent 6.3 software package, and the SIMPLE algorithm is used to resolve the coupling of both pressure and velocity. Furthermore, the userdefined scalar and user-defined function are loaded into Fluent6.3 to add the energy equation, and then, a twotemperature model is carried out.

\section{Numerical results and discussion}

\subsection{Flame front inclination instability}

Figure 2 shows the evolution of flame front inclination instability for the initial preheating temperature difference of $300 \mathrm{~K}$ at the inlet filtration velocity $u_{\mathrm{g}}=0.7$ $\mathrm{m} / \mathrm{s}$ and equivalence ratio $\varphi=0.4$. The magnitude of final inclinational angle is approximately $45^{\circ}$. This flame behavior is coincided well with Zheng's numerical 
results [4], but their igniting condition is an initial flame front with an inclinational angle of $36.25^{\circ}$ and ignition temperature of $1400 \mathrm{~K}$ in the preheating section. As a consequence, it is confirmed that the flame front instability could occur when the ignition section cannot be initially preheated well. Furthermore, it is also indicated that the initial preheating uniformity plays a significant part in the stability of combustion wave propagating downstream. In essence, the thermal perturbation is arisen due to the filtration field nonuniformity, which may be the filtration velocity, or the

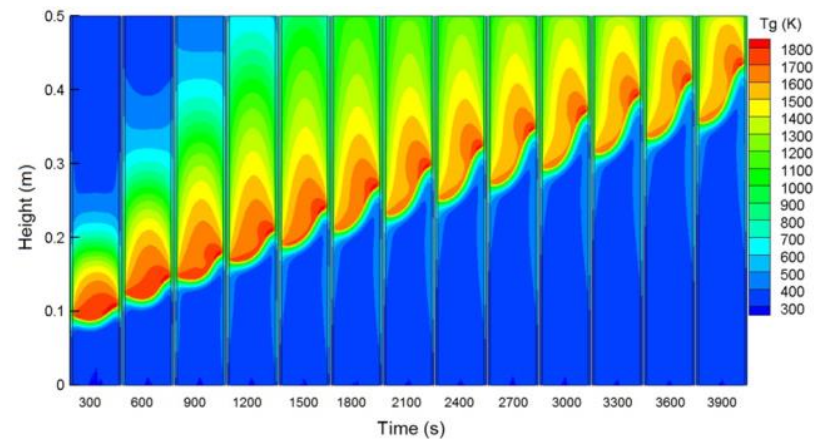

Fig. 2. The evolution of flame front inclination instability for initial preheating temperature difference of $300 \mathrm{~K}$ at $\mathrm{ug}_{\mathrm{g}}=0.7$ $\mathrm{m} / \mathrm{s}, \varphi=0.4$.

local porosity of packed bed. Further, it implies that the reaction front is not plannar at the beginning of combustion wave propagation.

When the combustion wave propagates downstream, the hydrodynamic instability [1,9] will dominate the evolution of flame front deformation. Most of the methane fuel flows to the right reaction zone, and consequently, the right flame temperature rises to approach nearly to the left flame temperature, or so much as in excess of the left temperature. At the later stage of wave propagation, when heat release in the reaction zone is in system thermal equilibrium state, the deformation behavior of flame front will no longer continue to develop.

Fig. 3 quantitatively presents the growth profiles of flame front inclinational angle with variation of preheating temperature differences at $\mathrm{u}_{\mathrm{g}}=0.7 \mathrm{~m} / \mathrm{s}, \varphi=0.4$. It shows that the magnitude of flame inclination instability increases depending on the increment of preheating temperature difference. When the initial thermal perturbation is large, the magnitude of inclinational angle grows rapidly and linearly at the early stage of inclination instability development, and it develops slowly to assume mostly horizontal-linearly at the later stage. However, when the preheating temperature difference is small below $100 \mathrm{~K}$, the growth profiles of inclinational angle assume like $\mathrm{S}$-shape. This instability development may be called as perturbation three-staged evolution.

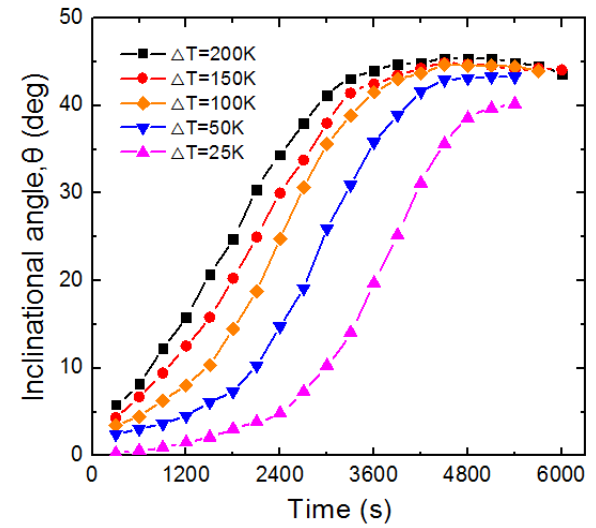

Fig. 3. Inclinational angle growth profiles for various initial preheating temperature differences at $\mathrm{ug}_{\mathrm{g}}=0.7 \mathrm{~m} / \mathrm{s}, \varphi=0.4$.

According to the relationship of combustion wave propagation velocity [10], the local wave propagation velocity can be used to analyze the evolution of flame front inclinational angle. At the beginning of wave propagation, the initial perturbation of preheating section is taken place. Consequently, the local propagation velocity is no longer uniform for the whole flame front. The difference between the left and right local wave velocities is amplified at the early stage, accordingly, the growth of the front inclinational angle increases rapidly. Nevertheless, at the later stage of wave propagation, as the chemical reaction approaches to equivalent at between the above front and below front. And thus, the local wave propagation velocities reach approximately consistent for the whole flame front. This indicates that the front inclinational angle approaches to a constant.

\subsection{Flame front break instability}

Fig. 4 shows that the effects of filtration velocity and equivalence ratio on the flame front instability with the initial preheating perturbation of $300 \mathrm{~K}$. It can be seen in Fig. 4(a) that the flame front is sharply stretched with appearance of inclination instability at the beginning stage of $900 \mathrm{~s}$, and at the propagating time of about 1200 $\mathrm{s}$, the flame front is divided into two high temperature zones, which may be called as the occurrence of the flame front break instability. As shown Fig. 4(b), when the filtration velocity increases to $u_{g}=0.8 \mathrm{~m} / \mathrm{s}$, the appearance time of front break instability is advanced to 900 s. It is also further demonstrated that the higher filtration velocity intensifies the development of flame front instability. 


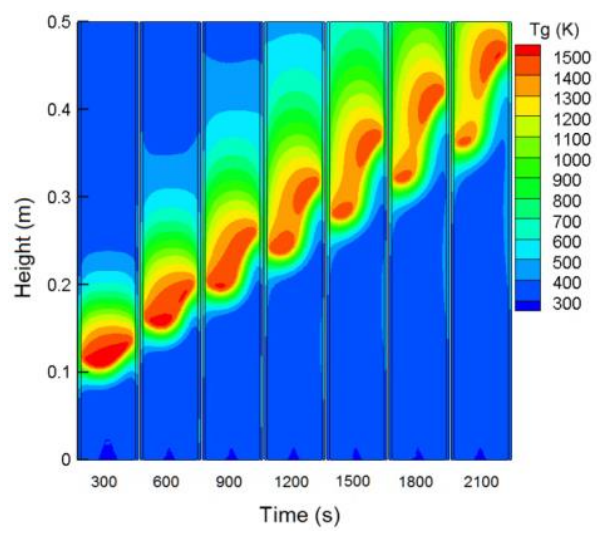

(a) $u_{\mathrm{g}}=0.6 \mathrm{~m} / \mathrm{s}$

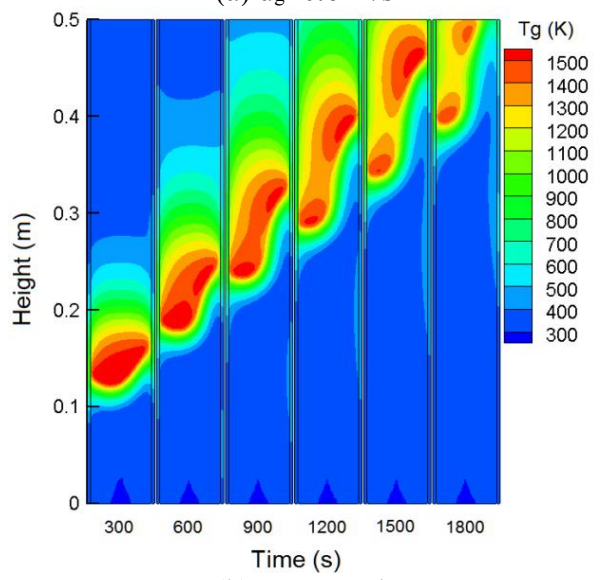

(b) $u_{\mathrm{g}}=0.8 \mathrm{~m} / \mathrm{s}$

Fig. 4. The evolutions of flame front break instability for initial preheating temperature difference of $300 \mathrm{~K}$ at (a) $u_{g}=0.6 \mathrm{~m} / \mathrm{s}$, $\varphi=0.2$, (b) $u_{\mathrm{g}}=0.8 \mathrm{~m} / \mathrm{s}, \varphi=0.2$.

Compared to the results in Fig. 2, it can be found that the evolution of front instability develops faster depending on the lower equivalence ratio with the same initial preheating perturbation. But above all, it may be deduced that, on the one hand, increasing of the initial preheating perturbation leads to the intensification of the flame distortion, on the other hand, the initial perturbation of $300 \mathrm{~K}$ may be regarded as the critical point of the instability transition between the front inclination and break. Besides, appearance of the crack on the flame front surface may be associated with development of the hydrodynamic instability. It is conceivable that the flame front evolution at higher filtration velocities supplies a possible evidence of formation of the front break observed in the experiments

\subsection{Flame front shifting instability}

When the filtration velocity increases to $1.0 \mathrm{~m} / \mathrm{s}$, and the initial preheating perturbation rises to $400 \mathrm{~K}$, the numerical appearance of flame front shifting instability at $\varphi=0.2$ is shown in Fig. 5. As seen in Fig. 5(a), the evolution of flame front shifting instability shows that the flame front gradually shrinks to assume the wave propagating path shifting to the left side of burner. To clearly analyze the development of the front shifting instability, the methane concentration contour is shown in Fig. 5(b). A gap of fuel leakage appears on the right side of burner. It means that the initial preheating extreme non-uniformity on the transverse of burner will lead to the incomplete combustion. With the combustion wave propagating downstream, the gap of fuel leakage gradually increases, and consequently, the more methane escapes.

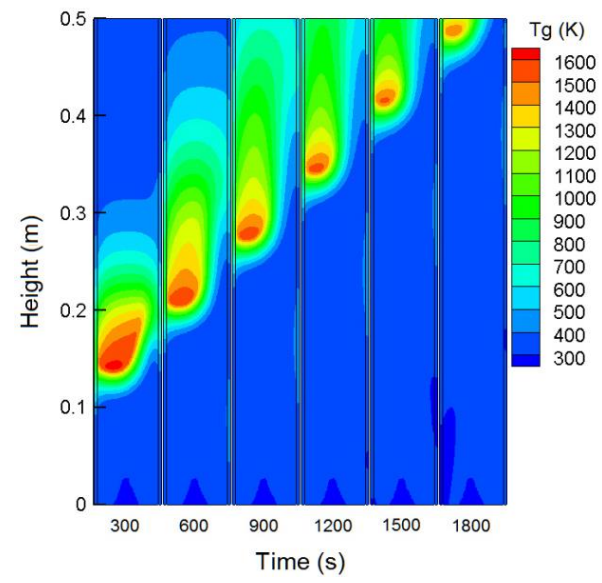

(a) temperature contour

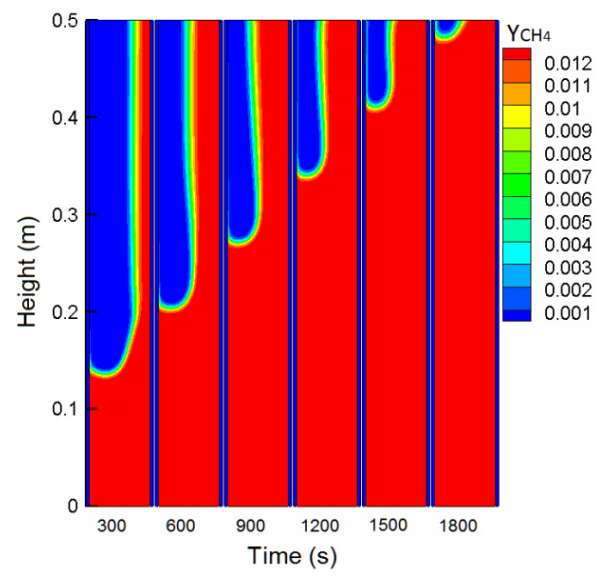

(b) $\mathrm{CH}_{4}$ mass fraction contour

Fig. 5. The evolution of flame front shifting instability for initial preheating temperature difference of $400 \mathrm{~K}$ at $\mathrm{ug}_{\mathrm{g}}=1.0$

$$
\mathrm{m} / \mathrm{s}, \varphi=0.2 \text {. }
$$

Due to the initial partial igniting, the heat release in the reaction zone is not enough to sustain the thermal equilibrium of combustion. As a portion of the heat release in the reaction zone is carried away with the escaped gas mixture, and overall heat losses is continuously increased, the essential igniting energy is reduced to result in the reaction zone shrinking gradually during the wave propagating downstream. Furthermore, increasing of filtration velocity will lead to the intensification of hydrodynamic instability. This highly developed hydrodynamic instability will further aggravate the shrink of flame front size. Therefore, the development of flame front shifting instability may be deemed to be simultaneously affected by the perturbations both of the thermal non-equilibrium and the hydrodynamic instability. 


\section{Conclusions}

The effects of the initial preheating perturbation on the dynamical behaviors of FGC wave propagation instability for low-velocity FGC in packed bed are studied numerically. The typical flame shapes are obtained depending on filtration velocity, equivalence ratio, and initial preheating temperature difference. It is demonstrated that the development of flame front inclination instability is proportional to the magnitude of initial preheating perturbation. At lower equivalence ratio, the initial preheating perturbation of $300 \mathrm{~K}$ leads to the evolution of flame front break. Increasing of filtration velocity brings forward the appearance of flame front break, due to the intensification of the hydrodynamic instability. In addition, a new instability of flame front shifting is also confirmed with the initial thermal perturbation of $400 \mathrm{~K}$, which results in a fuel leakage of incomplete combustion.

\section{References}

1. S.S. Minaev, S.I. Potytnyakov, V.S. Babkin, Combust. Explos. Shock Waves 30 (1994) 761-763.
2. K.V. Dobrego, S.A. Zhdanok, A.I. Zaruba, Int. J. Heat Mass Transfer 44 (2001) 2127-2136.

3. K.V. Dobrego, I.M. Kozlov, V.I. Bubnovich, C.E. Rosas, Int. J. Heat Mass Transfer 46 (2003) 32793289.

4. C.H. Zheng, L.M. Cheng, A. Saveliev, Z.Y. Luo, K.F. Cen, Int. J. Heat Mass Transfer 54 (2011) 3642-3649.

5. J.R. Shi, M.Z. Xie, Z.J. Xue, Y.N. Xu, H.S. Liu, Int. J. Heat Mass Transfer 55 (2012) 7063-7071.

6. N. Wakao, S. Kaguei, Heat and Mass Transfer in Packed Beds, Gordon and Breach Science Publications, New York, 1982.

7. A.J. Barra, J.L. Ellzey, Combust. Flame 137 (2004) 230-241.

8. V. Bubnovich, M. Toledo, Appl. Therm. Eng. 27 (2007) 1144-1149.

9. C.K. Law, Combustion Physics, Cambridge University Press, New York, 2006.

10. S. Zhdanok, L.A. Kennedy, G. Koester, Combust. Flame $\quad \mathbf{1 0 0} \quad$ (1995) 221-231. 